\title{
A method of estimating the contribution factors of a sound source, using envelopes of band-passed signals
}

\author{
Tsutomu Itoh, ${ }^{*}$ Hiroyuki Noto, ${ }^{* *}$ Masato Abe, ${ }^{* * *}$ and Ken'iti Kido** \\ * $R$ \& D Division, Magnetic Products Group, SONY Corp., \\ 3-4-1, Sakuragi, Tagajo, 985 Japan \\ **Research Center for Applied Information Sciences, Tohoku University, \\ 2-1-1, Katahira, Sendai, 980 Japan \\ ***Education Center for Information Processing, Tohoku University, \\ Kawauchi, Sendai, 980 Japan
}

(Received 29 September 1983)

\begin{abstract}
This paper describes a method of estimating sound source contribution factors; it utilizes envelopes of band-passed signals picked up at the noise source and at the observation point. The coherence function has been used to express the contribution factor of a source signal to an observation point signal. In the open air, fluctuation of the transmission time caused by wind and/or air turbulence decreases the value of the coherence function; in other words the estimated transfer characteristics contain considerable error. The effect of such fluctuations increases in proportion to the length of transmission path and the frequency of signals. Thus, the authors propose in this paper the use of envelopes of band-passed signals instead of the original wave forms for estimating sound source contribution factors. With the cross spectral technique applied to those envelopes, the power transmission rate and the contribution factor of each frequency band can be obtained without being affected by a fluctuation of the transmission time. The method proposed here is useful for noise source detection.
\end{abstract}

PACS number: 43. 50. Yw, 43. 60. $-\mathrm{c}$

\section{INTRODUCTION}

In noise measurement the contribution factor is known to be one of the most useful parameters. It is defined as the ratio of the noise power at an observation point of a specified source to the total power. Commonly used in estimating the contribution factor is the ratio of the noise power at an observation point plus the noise from a specified source to that without it. However, since it is often impossible to turn on and off the source noise independently of the others, this method is not always practical.

The coherence function calculated by the cross spectral technique is well known as being able to indicate the contribution factor of a source signal to a noise at an observation point. The function can be derived from the power spectra and cross spectrum without turning on and off the source noise. However, it is often useless when the transmission path between the source and the observation point is long and transmission time varies because of wind and/ or air turbulence. ${ }^{1,2)}$ The effect of a long transmission time can be removed by shifting the time window for the observation point signal forward from the location of the window for the source signal. The shift time can be measured either by using the crosscorrelation between the source signal and the signal at the observation point or by using the length of the transmission path. However, it is rather hard to remove the effect of the fluctuation of transmission time because the cross spectral technique is based on the assumption that the noise transmission system is time-invariant.

In order to resolve this difficulty, this paper pro- 
poses a new method using the envelopes of signals. ${ }^{3)}$ With the cross spectral technique applied to envelopes of band-passed signals instead of to the original signal, the power transmission rate and the contribution factor of the source can be estimated without significant error as a function of the frequency band. The proposed method is adequate specifically for an estimation of the contribution factor using an actual noise.

It will be shown first that the measurement of transfer characteristics of a noise transmission system using the conventional cross spectral technique contains considerable error. The method using envelopes of band-passed signals will then be presented. After explaining the two techniques of extracting the envelope signal the results of a computer simulation and an experiment in the open air will be described.

\section{CONTRIBUTION FACTOR MEASUREMENT USING THE COHERENCE FUNCTION}

The coherence function is applied to noise source identification, measurement of signal-to-noise power ratio, error analysis for transfer function measurement and a directive spectrum analysis. ${ }^{4-6)}$ In noise measurement, it is often used for estimating the contribution factor of a noise source at an observation point.

A noise transmission system in the open air is shown in Fig. 1, which has $n$ noise sources $S_{i}(i=1$, $\ldots, n)$ and an observation point $M$. The transfer function $H_{i}(f)(i=1, \ldots, n)$ and the coherence function $\gamma_{i y}{ }^{2}(f)$ between source signal $x_{i}(t)$ and signal $y(t)$ at $\mathrm{M}$ are defined as

$$
H_{i}(f)=W_{i y}(f) / W_{i i}(f)
$$

and

$$
\begin{aligned}
\gamma_{i y}{ }^{2}(f) & =\left|W_{i y}(f)\right|^{2} / W_{i i}(f) W_{y y}(f) \\
& =\left|H_{i}(f)\right|^{2} W_{i i}(f) / W_{y y}(f),
\end{aligned}
$$

where $W_{i i}(f)$ and $W_{y y}(f)$ are the power spectra of $x_{i}(t)$ and $y(t)$, respectively, and $W_{i y}(f)$ is the cross spectrum of $x_{i}(t)$ and $y(t)$. It is clear from Eq. (2) that the coherence function expresses the contribution factor of source $x_{i}(t)$ at observation point $\mathrm{M}$. Therefore, if the transmission path is linear and timeinvariant and the transmission time is negligibly short compared with the time window length, it appears that

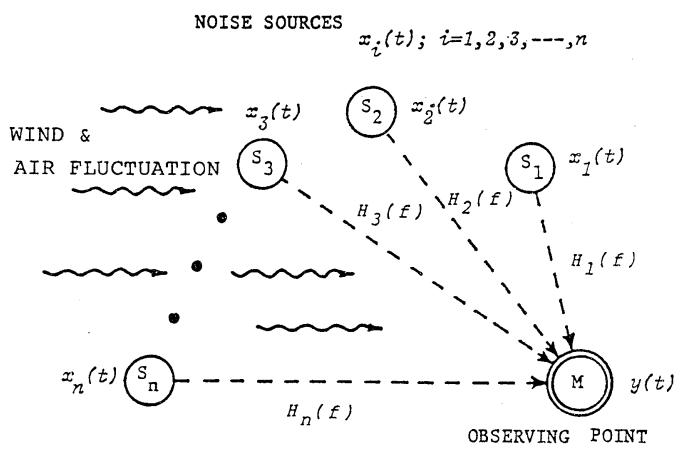

Fig. 1 Noise transmission system in the open air with the wind and the air turbulence.
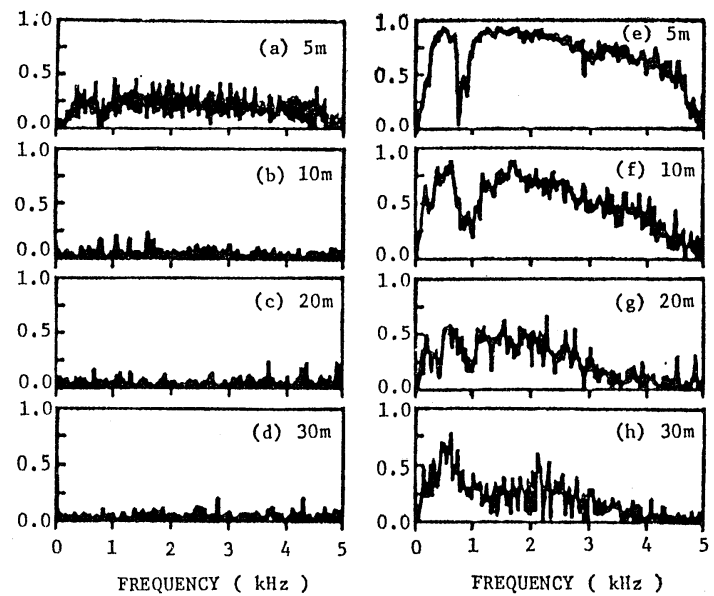

Fig. 2 Coherence function between the source signal and the observation point signal measured in the open air, with the application of the conventional cross spectral technique. $(a) \sim(d)$ : without the shift of the time window, (e) $\sim(\mathrm{h})$ : with the shift of the time window due to the transmission time.

$$
\sum_{i=1}^{n} \gamma_{i y}{ }^{2}(f)=1.0 .
$$

In open air measurement, the transmission time of a system is long and frequently varied due to wind and/or air turbulence. Consequently, the estimated coherence function decreases its magnitude and includes a considerable error.

Figure 2 shows the effects of a long and varying transmission time (the estimated coherence functions between the source signal of a white noise and the 


\section{T. ITOH et al.: CONTRIBUTION FACTOR ESTIMATION USING ENVELOPES}

observation point signal in the field). The observation points were set at $5,10,20$ and $30 \mathrm{~m}$ from the source. The average wind velocity was about $2 \mathrm{~m} /$ s. The background noise level was about $50 \mathrm{dBA}$ and the source noise level was set at about $60 \mathrm{dBA}$ at the each observation point.

Figures 2 (a) (d) are the results in which the time window compensation due to the transmission time was not given. Since the length of the time window was $51.2 \mathrm{~ms}$, which was too short compared with the transmission time, the estimated coherence function was much smaller than the expected value 0.9 , which is derived from the source noise level and the background noise level. On the other hand, the coherence functions which were calculated with the shift of the time window due to the transmission time are shown in Figs. 2 (e) $\sim(\mathrm{h})$. As the time window compensation removed the effect of the fluctuating transmission time, the coherence function increased to about 0.9 in the case where the transmission path was $5 \mathrm{~m}$. However, the estimated value decreased as the transmission path became longer and the frequency of the signal higher. The reason for this is that the fluctuation of the phase shift due to the varying transmission time becomes greater in proportion to the length of the path and the frequency of the source signal.

It is found from the experiment that the coherence function computed by the conventional cross spectral technique is often useless for contribution factor estimation in field noise measurement.

\section{CONTRIBUTION FACTOR MEASUREMENT USING ENVELOPES}

A new method of contribution factor measurement is proposed. In this method the cross spectral technique is applied to envelope signals extracted from the low frequency components of the squared signals instead of the original signals. The power transmission rate and the contribution factor of the frequency band to be estimated can be obtained by use of the envelopes of the output of the band-pass filter.

In the first place, it is assumed that the observation point signal consists of signals from plural noise sources which are mutually uncorrelated. And, it is indicated that the cross spectrum between the fluctuating components of the envelopes of the source and the observation point signal can be calculated without being affected by another source, and that the power transmission rate can be obtained accurately.

Here, if $x_{i}(t)$ and $y(t)$ are the band-pass filter output signals of source $i$ and the observation point signal, respectively, and have a finite duration, the Fourier transforms can be defined as $X_{i}(f)$ and $Y(f)$. The squared signals of $x_{i}(t)$ and $y(t)$ are $\hat{x}_{i}(t)$ and $\hat{y}(t)$, respectively, and $\hat{X}_{i}(\hat{f})$ and $\hat{Y}(\hat{f})$ are the Fourier transforms of $\hat{x}_{i}(t)$ and $\hat{y}(t)$, respectively. And if the band-pass filter width is set at from $f_{1}$ to $f_{2}$ and $2 f_{1}>$ $\left(f_{2}-f_{1}\right)$, the squared signals of the outputs contain the differential and additional components and these components can be separated from each other. It will be found that the differential component, which has the frequency band from $f_{1}-f_{2}$ to $f_{2}-f_{1}$, is useful for our method.

Since squaring in the time domain is equivalent to the convolution integral in the frequency domain, $\hat{X}_{i}(\hat{f})$ and $\hat{Y}(\hat{f})$ are described as

$$
\hat{X}_{i}(\hat{f})=\int_{-\infty}^{\infty} X_{i}(f) X_{i}(\hat{f}-f) d f
$$

and

$$
\hat{Y}(\hat{f})=\int_{-\infty}^{\infty} Y(f) Y(\hat{f}-f) d f .
$$

The power spectrum $\hat{W}_{i i}(\hat{f})$ of $\hat{x}_{i}(t)$ and the cross spectrum $\hat{W}_{i y}(\hat{f})$ of $\hat{x}_{i}(t)$ and $\hat{y}(t)$ are described as follows.

$$
\begin{aligned}
\hat{W}_{i i}(\hat{f})= & E\left[\hat{X}_{i}(\hat{f}) \hat{X}_{i}^{*}(\hat{f})\right] \\
= & E\left[\int_{-\infty}^{\infty} X_{i}(f) X_{i}(\hat{f}-f) d f\right. \\
& \left.\cdot \int_{-\infty}^{\infty} X_{i}^{*}(f) X_{i}^{*}(\hat{f}-f) d f\right]
\end{aligned}
$$

and

$$
\begin{aligned}
\hat{W}_{i y}(\hat{f})= & E\left[\hat{Y}(\hat{f}) \hat{X}_{i}^{*}(\hat{f})\right] \\
= & E\left[\int_{-\infty}^{\infty} Y(f) Y(\hat{f}-f) d f\right. \\
& \left.\cdot \int_{-\infty}^{\infty} X_{i}^{*}(f) X_{i}^{*}(\hat{f}-f) d f\right],
\end{aligned}
$$

where $E[\quad]$ and * donote the expected value and the complex conjugate, respectively. If the transfer function between source $i$ and the observation point is described as $H_{i}(f)+N_{i}(f)$, where $H_{i}(f)$ is the linear and time-invariant component, and $N_{i}(f)$ is the component confused by wind and/or turbulence, $Y(f)$ is expressed as

$$
Y(f)=\sum_{i=1}^{n}\left\{H_{i}(f)+N_{i}(f)\right\} X_{i}(f) .
$$


With Eq. (8) $\hat{W}_{i y}(\hat{f})$ is described as

$$
\begin{aligned}
\hat{W}_{i y}(\hat{f})= & E\left[\int_{-\infty}^{\infty}\left\{\sum_{k=1}^{n}\left(H_{k}(f)+N_{k}(f)\right) X_{k}(f)\right\}\right. \\
& \cdot\left\{\sum_{l=1}^{n}\left(H_{l}(\hat{f}-f)+N_{l}(\hat{f}-f)\right) X_{l}(\hat{f}-f)\right\} d f \\
& \left.\times \int_{-\infty}^{\infty} X_{i}^{*}(f) X_{i}^{*}(\hat{f}-f) d f\right] \\
= & E\left[\int _ { - \infty } ^ { \infty } \sum _ { k = 1 } ^ { n } \sum _ { l = 1 } ^ { n } \left\{\left(H_{k}(f) H_{l}(\hat{f}-f)\right.\right.\right. \\
& +N_{k}(f) H_{l}(\hat{f}-f)+H_{k}(f) N_{l}(\hat{f}-f) \\
& \left.\left.+N_{k}(f) N_{l}(\hat{f}-f)\right) X_{k}(f) X_{l}(\hat{f}-f)\right\} d f \\
& \left.\times \int_{-\infty}^{\infty} X_{i}^{*}(f) X_{i}^{*}(\hat{f}-f) d f\right] .
\end{aligned}
$$

Since $N_{k}(f)$ is a random variable, $E\left[N_{k}(f) H_{l}(\hat{f}-f)\right]$ and $E\left[H_{k}(f) N_{l}(\hat{f}-f)\right]$ are equal to zero. And $N_{k}(f)$ and $N_{l}(f)$ are uncorrelated with each other, so $E\left[N_{k}(f) N_{l}(\hat{f}-f)\right]$ can be neglected in the case where $0<|\hat{f}|<2 f_{1}$. Therefore, Eq. (9) is rewritten as

$$
\begin{aligned}
\hat{W}_{i y}(\hat{f})= & E\left[\int _ { - \infty } ^ { \infty } \left\{\sum_{k=1}^{n} \sum_{l=1}^{n} H_{k}(f) H_{l}(\hat{f}-f)\right.\right. \\
& \left.\cdot X_{k}(f) X_{l}(\hat{f}-f)\right\} d f \\
& \left.\times \int_{-\infty}^{\infty} X_{i}^{*}(f) X_{i}^{*}(\hat{f}-f) d f\right] \\
& \left(\because \quad 0<|\hat{f}|<2 f_{1}\right) .
\end{aligned}
$$

The noise sources are uncorrelated with each other, so the expected value $X_{k}(f) X_{l}(\hat{f}-f)$ can be neglected in the case where $k \neq l$. Thus, the following equation is obtained:

$$
\begin{aligned}
\hat{W}_{i y}(\hat{f})= & E\left[\int _ { - \infty } ^ { \infty } \left\{\sum_{k=1}^{n} H_{k}(f) H_{k}(\hat{f}-f)\right.\right. \\
\cdot & \left.X_{k}(f) X_{k}(\hat{f}-f)\right\} d f \\
& \left.\times \int_{-\infty}^{\infty} X_{i}^{*}(f) X_{i}^{*}(\hat{f}-f) d f\right] \\
& \left(\because 0<|\hat{f}|<2 f_{1}\right) .
\end{aligned}
$$

Here, it is assumed that the transfer function $H_{i}(f)$ is expressed in terms of the constant transfer rate $\alpha_{i}$ and the transmission delay time $\tau_{i}$ as

$$
H_{i}(f)=\alpha_{i} \cdot e^{-j 2 \pi f \tau_{i}} .
$$

Since this assumption is required to hold only in the narrow band to be measured, it can be accepted easily. Therefore, the following equation is also accepted:

$$
\begin{aligned}
H_{k}(f) H_{k}(\hat{f}-f) & =\alpha_{k} e^{-j 2 \pi f \tau_{k}} \cdot \alpha_{k} e^{-j 2 \pi(\hat{f}-f) \tau_{k}} \\
& =\alpha_{k}^{2} e^{-j 2 \pi \hat{f}_{k}} .
\end{aligned}
$$

By substituting Eq. (13) in Eq. (11), $\hat{W}_{i y}(\hat{f})$ can be rearranged and described as

$$
\begin{aligned}
\hat{W}_{i y}(\hat{f})= & E\left[\int_{-\infty}^{\infty}\left\{\sum_{k=1}^{n} \alpha_{k}^{2} e^{-j 2 \pi \hat{f} \tau_{k}} X_{k}(f) X_{k}(\hat{f}-f)\right\} d f\right. \\
& \left.\times \int_{-\infty}^{\infty} X_{i}^{*}(f) X_{i}^{*}(\hat{f}-f) d f\right] \\
= & \sum_{k=1}^{n}\left\{\alpha_{k}^{2} e^{-j 2 \pi \hat{f}_{k}}\right. \\
& \cdot E\left[\int_{-\infty}^{\infty} X_{k}(f) X_{k}(\hat{f}-f) d f\right. \\
& \left.\left.\cdot \int_{-\infty}^{\infty} X_{i}^{*}(f) X_{i}^{*}(\hat{f}-f) d f\right]\right\} \\
& \left(\because \quad 0<|\hat{f}|<2 f_{1}\right) .
\end{aligned}
$$

Since the noise sources are uncorrelated with each other, the squared signals are also uncorrelated with each other except for the component at $\hat{f}=0$ and $\hat{f}=2 f$, and the expected value can be neglected in the case where $0<|\hat{f}|<2 f_{1}$ and $k \neq i$. Consequently $\hat{W}_{i y}(\hat{f})$ is expressed as

$$
\begin{aligned}
\hat{W}_{i y}(\hat{f})= & \alpha_{i}{ }^{2} e^{-j 2 \pi \hat{f}_{i}} \\
& \cdot E\left[\int_{-\infty}^{\infty} X_{i}(f) X_{i}(\hat{f}-f) d f\right. \\
& \left.\cdot \int_{-\infty}^{\infty} X_{i}{ }^{*}(f) X_{i}{ }^{*}(\hat{f}-f) d f\right] \\
= & \alpha_{i}{ }^{2} e^{-j 2 \pi \hat{f}_{i}} \cdot \hat{W}_{i i}(\hat{f}) \\
& \left(\because 0<|\hat{f}|<2 f_{1}\right) .
\end{aligned}
$$

From Eq. (15), the squared value $\alpha_{i}^{2}$, which indicates the power transmission rate from the source to the observation point, is expressed as

$$
\begin{gathered}
\alpha_{i}{ }^{2}=\left|\hat{W}_{i y}(\hat{f})\right| / \hat{W}_{i i}(\hat{f}) \\
\left(\because \quad 0<|\hat{f}|<2 f_{1}\right) .
\end{gathered}
$$

Since the components at $\hat{f}=0$ of $\hat{X}_{i}(\hat{f})$ and $\hat{Y}(\hat{f})$ are the average values of the squared signals $\hat{x}_{i}(t)$ and $\hat{y}(t)$, respectively, the contribution factor $C F_{i}$ of the $i$-th source using the coefficient $\alpha_{i}{ }^{2}$ is represented as 


\section{T. ITOH et al.: CONTRIBUTION FACTOR ESTIMATION USING ENVELOPES}

$$
C F_{i}=\alpha_{i}{ }^{2} E\left[\hat{X}_{i}(0)\right] / E[\hat{Y}(0)] .
$$

However, the estimated value $\alpha_{i}^{2}$ contains errors which are statistically caused by the finite averaging times and caused by the unreasonableness of the assumption of Eq. (12). That is, $\alpha_{i}{ }^{2}$ changes its value with a frequency change. In the new method, in order to decrease such errors, the correct power transmission rate $A_{i}{ }^{2}$ is introduced, which is calculated from the weighted average of $\alpha_{i}{ }^{2}(\hat{f})$ in the low frequency range. $\alpha_{i}{ }^{2}(\hat{f})$ is the estimated power transmission rate and is a function of $\hat{f}$ defined as

$$
\begin{gathered}
\alpha_{i}{ }^{2}(\hat{f})=\left|\hat{W}_{i y}(\hat{f})\right| / \hat{W}_{i i}(\hat{f}) \\
\left(\because \quad 0<|\hat{f}|<2 f_{1}\right) .
\end{gathered}
$$

$A_{i}{ }^{2}$ is expressed as

$$
A_{i}{ }^{2}=\int_{f_{3}}^{f_{4}} \alpha_{i}{ }^{2}(\hat{f}) \hat{W}_{i i}(\hat{f}) d \hat{f} / \int_{f_{3}}^{f_{4}} \hat{W}_{i i}(\hat{f}) d \hat{f} .
$$

In Eq. (19), $f_{3}$ is selected to be a frequency in the vicinity of zero, so as to avoid the effect of the sidelobe of the DC component, and $f_{4}$ is lower than $2 f_{1}$ and set low enough to avoid the effect of wind and/ or air turbulence. Thus, the contribution factor $C F_{i}$ is represented as

$$
C F_{i}=A_{i}{ }^{2} E\left[\hat{X}_{i}(0)\right] / E[\hat{Y}(0)] .
$$

The contribution factor can be obtained using the low frequency components of the squared signals, which this paper calls the envelope.

Shown in Fig. 3 is the flowchart of estimating the contribution factors using envelopes. By use of this method, noise measurement can be carried out without being affected by wind and/or air turbulence, and the contribution factor can be obtained as a function of each pass band. The pass band width of the band-pass filter can be selected arbitrarily, but it should be emphasized that an excessively narrow band width should not be chosen if the contribution factor is to be obtained as a function of a small frequency step. The reason for this is the possibility that the components in the frequency band are frequency-modulated by the varying transmission time and, as a result, fail to stay within the specified band. It seems that the $1 / 3$ octave band width is appropriate for ordinary noise measurement.

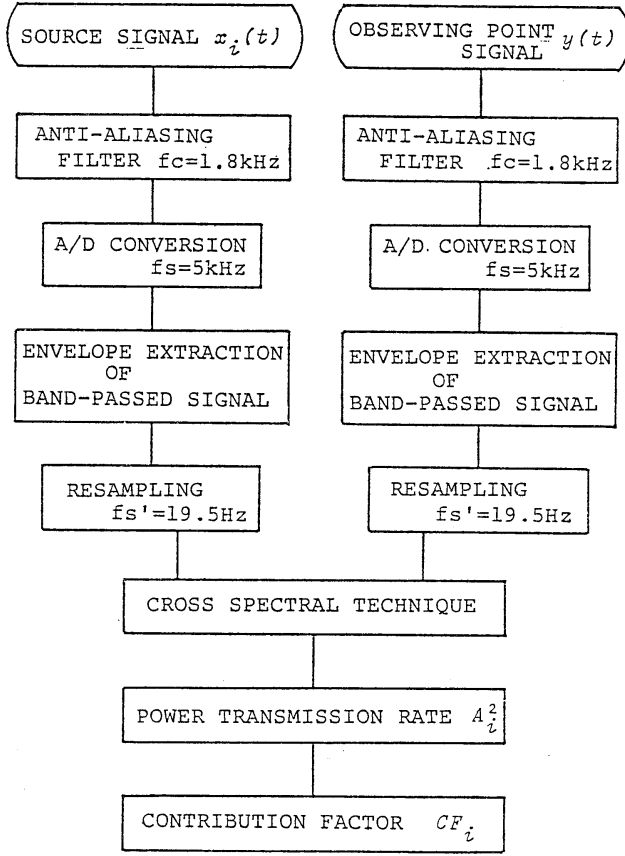

Fig. 3 Flowchart of the method of estimating the contribution factor using the envelopes of the band-passed signals.

\section{METHOD OF DETECTING THE ENVELOPE OF THE BAND-PASSED SIGNAL}

The envelope is derived from the low frequency component of the squared signal of the output of the band-pass filter. There are two methods of detecting the envelope. One uses a digital band-pass filter, whereas the other uses DFT's (discrete Fourier transforms) multiplied by the frequency response of the band-pass filter. Prior to the explanation of these two methods, the band-pass filter characteristics are indicated here. The 4th-order Tchebycheff filter of the $1 / 3$ octave band width is used, which is obtained using a bi-linear transformation. The band-pass filter characteristics are shown in Fig. 4. Each frequency band is described in Table 1.

The procedure of the method using the digital band-pass filter, called the filter method in this paper, is shown in Fig. 5. The A/D-converted signal is fed to the desired band-pass filter; the output from the filter is squared; the envelope can be extracted from the low frequency component of the squared signal. The FIR digital low-pass filter with Hanning char- 
Table 1 Pass band frequencies of the $1 / 3$ octave band-pass filter bank used for the experiment.

\begin{tabular}{lcc}
\hline & $\begin{array}{c}\text { Pass band } \\
\text { frequency }(\mathrm{Hz})\end{array}$ \\
\hline Filter 1 & $224 \sim 283$ \\
Filter 2 & $283 \sim 356$ \\
Filter 3 & $356 \sim 449$ \\
Filter 4 & $449 \sim 566$ \\
Filter 5 & $566 \sim 713$ \\
Filter 6 & $713 \sim 898$ \\
Filter 7 & $898 \sim 1,130$ \\
Filter 8 & $1,130 \sim 1,430$ \\
Filter 9 & $1,430 \sim 1,800$ \\
Filter 10 & $1,800 \sim 2,260$ \\
\hline
\end{tabular}

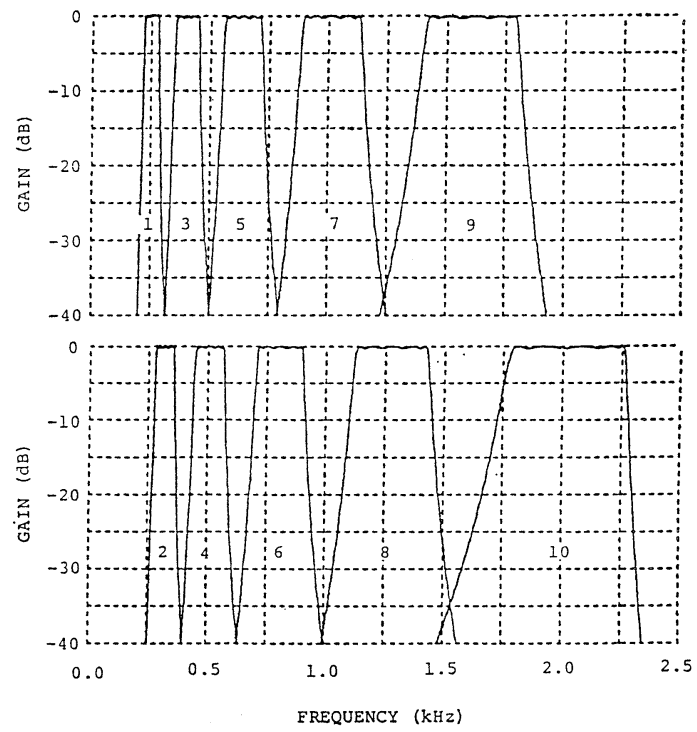

Fig. 4 Frequency responses of the $1 / 3$ octave band-pass filter bank used in the experiments.

acteristics is used to obtain the low frequency component. The envelope sequence is sampled again with a lower frequency and is used for the data of the cross spectral technique.

The procedure using the DFT's, called the DFT method, is shown in Fig. 6. The sequence cut out from the original data is discrete Fourier transformed and the squared output is calculated from the total power of the DFT's multiplied by the frequency response of the desired band-pass filter, as in Fig. 4. The squared sequence is calculated by shifting the

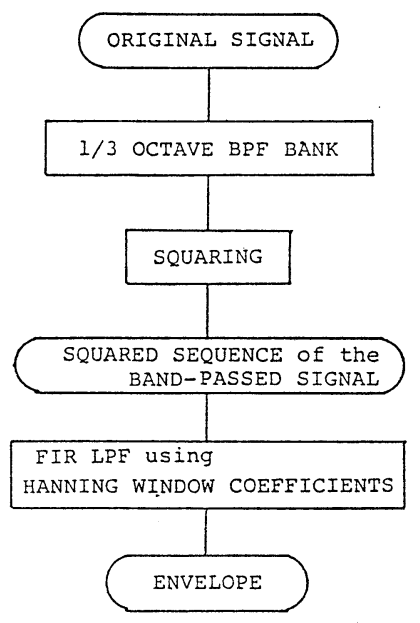

Fig. 5 Filter method of extracting the envelope of the band-passed signal.

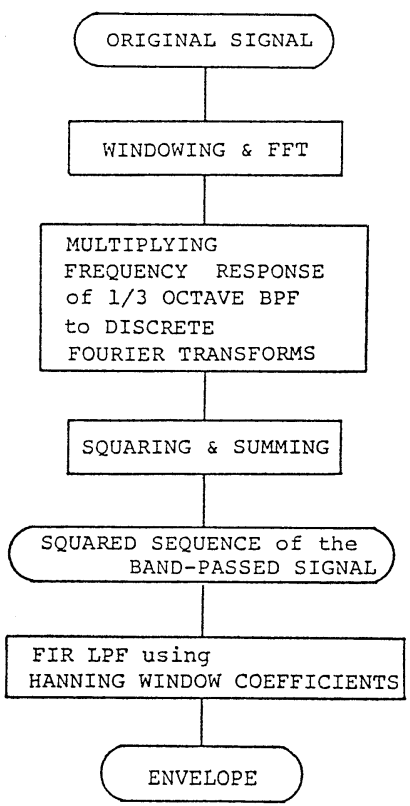

Fig. 6 DFT method of extracting the envelope of the band-passed signal.

time window which cuts out the data from the original. The envelope can be obtained, as in the filter method, from resampling the output of the same FIR digital low-pass filter.

Both of the above two methods of detecting the envelope have advantages and disadvantages. If the estimations of only a few bands are needed, the filter method is more useful than the DFT method. 


\section{T. ITOH et al.: CONTRIBUTION FACTOR ESTIMATION USING ENVELOPES}

On the contrary, the DFT method is useful in the case of multiple band estimation and is easily applied to narrow band estimation. Since the filter method contains the procedures which can be replaced by the analog technique, it is utilizable for portable and small system measuring of the contribution factor in the field. Both methods, which will be found equivalent to each other, can be used to derive the envelope.

\section{COMPUTER SIMULATION}

In this section, the results of a computer simulation are presented to indicate that the contribution factor using the envelopes is equivalent to that using the coherence function in the case of the linear and time-invariant system. The simulation was carried out under the condition that the transmission system had three sources and the transfer function $H_{i}(k)$ $(i=1,2,3)$ between the $i$-th source and the observation point was equal to 1.0. A human voice, music and an automobile noise were utilized as the source noises; they were mixed in the computer. The mixed signal was used as the observation point signal. The contribution factor was calculated in each $1 / 3$ octave band.

The contribution factor using the filter method and the DFT method are shown in Figs. 7 and 8, respectively. The horizontal axis represents the center frequency of the measuring $1 / 3$ octave band and the vertical axis represents the estimated contribution factor of each band. In calculating the contribution factor, $9.75 \mathrm{~Hz}$ was used as $f_{4}$ in Eq. (19). From the similar results obtained in both figures, it is observed

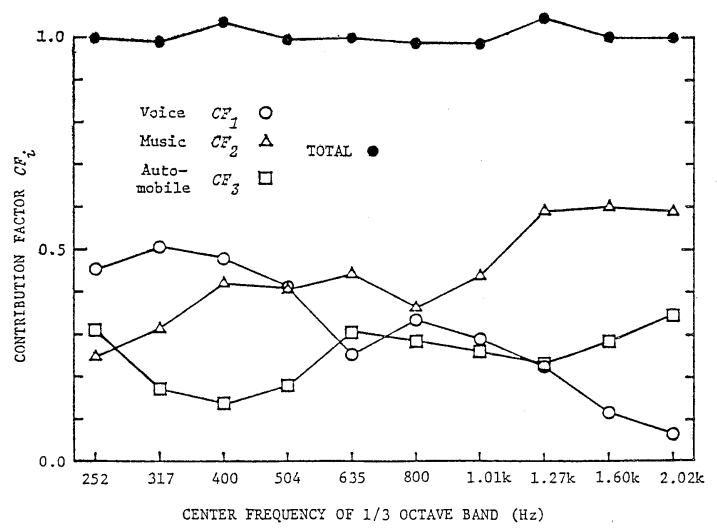

Fig. 7 Estimated contribution factor $C F_{i}$ of each $1 / 3$ octave band by the computer simulation using the filter method. that the two methods of detecting the envelope are equivalent. Since the total of the contribution factor of each source was about 1.0 , it is proved experimentally that the cross spectral technique can be applied to envelopes.

The contribution factors calculated from the coherence function applied to the original signal are shown in Fig. 9. The $C F_{i b}$ of the $1 / 3$ octave band $b$ was calculated as follows;

$$
\begin{gathered}
C F_{i \mathrm{~b}}=\int_{0}^{\infty} \gamma_{i y}{ }^{2}(f)\left|H_{\mathrm{b}}(f)\right|^{2} W_{y y}(f) d f \\
/ \int_{0}^{\infty}\left|H_{\mathrm{b}}(f)\right|^{2} W_{y y}(f) d f,
\end{gathered}
$$

where $H_{\mathrm{b}}(f)$ represents the frequency response of the

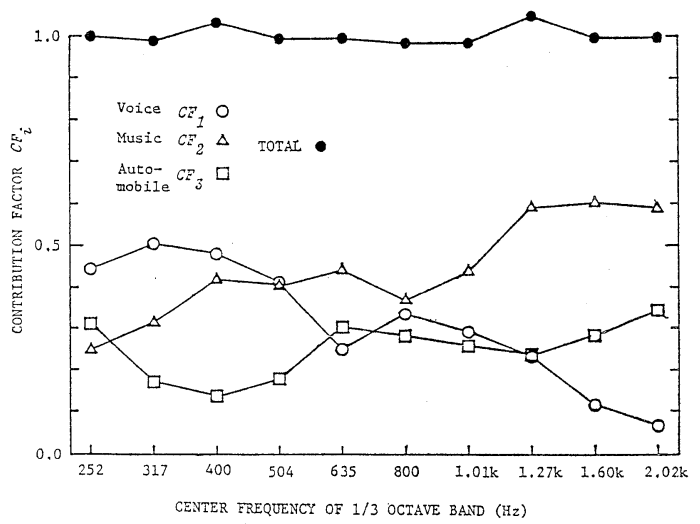

Fig. 8 Estimated contribution factor $C F_{i}$ of each $1 / 3$ octave band by the computer simulation using the DFT method.

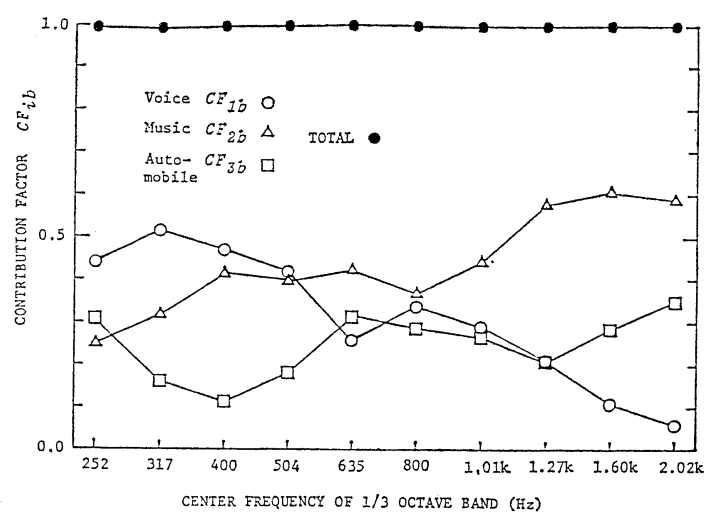

Fig. 9 Estimated contribution factor $C F_{i \text { b }}$ of each $1 / 3$ octave band by the computer simulation using the coherence function of the original signals. 


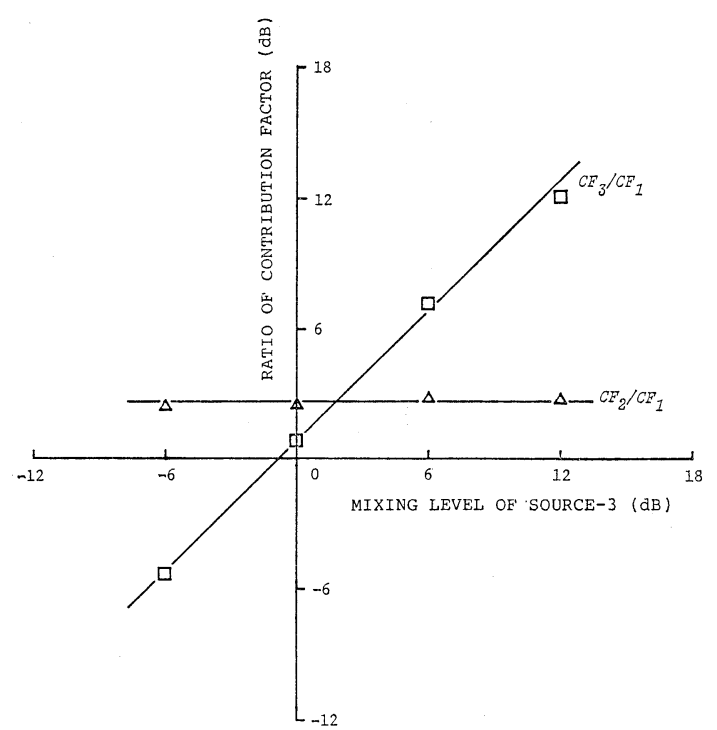

Fig. 10 Ratios of the contribution factors $C F_{2}$ and $C F_{3}$ to $C F_{1}$ versus the mixing level of the source 3 .

band-pass filter in Fig. 4 and Table 1. The $C F_{i_{\mathrm{b}}}$ in Fig. 9 was equal to that in Figs. 7 and 8.

In addition to the above simulation, a further experiment was carried out using the output signals from filter 8 in Table 1 of three white noises uncorrelated with each other. As shown in Fig. 10, the rates $C F_{3} / C F_{1}$ and $C F_{2} / C F_{1}$ of the contribution factors were calculated by varying the mixing level of source 3 in steps of $6 \mathrm{~dB} . C F_{2} / C F_{1}$ kept a constant value and $C F_{3} / C F_{1}$ was varied in steps of $6 \mathrm{~dB}$ due to the change in the mixing level. In this computer simulation, it is found that the contribution factor estimation using envelopes is both theoretically reasonable and experimentally justified.

\section{MEASUREMENT IN THE OPEN AIR}

In order to show that the present method was effective enough, an experiment in the open air was carried out. The noise transmission system had three noise sources; the sounds from them were observed at a point $30 \mathrm{~m}$ away as shown in Fig. 11; the human voice, the music and the automobile noise which were the same as those in the previous section were again used; each source level was set at about $60 \mathrm{dBA}$ at the observation point. The weather conditions and the background noise level were the same as in Fig. 2. The contribution factor of each source and each $1 / 3$ octave band is shown in Fig. 12. These results were obtained by use of the filter method. More reasonable results than those in Fig. 2 were obtained in each frequency band. Through the experiment, it is again verified that the method using envelopes is useful and powerful for noise source measurement.

\section{CONCLUSIONS}

The validity of a new method of estimating contribution factors was presented. Using the cross spectral technique applied to the envelopes of bandpassed signals, the contribution factor as a function of the frequency band is obtained without being

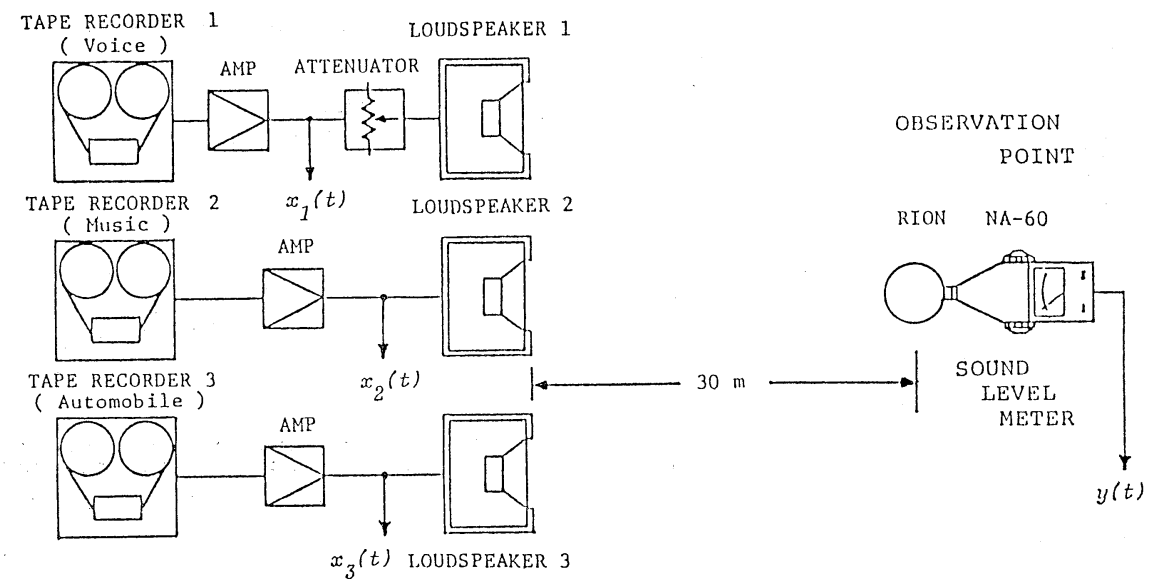

Fig. 11 Measurement system of the contribution factor of each source signal at the observation point in the open air. 


\section{T. ITOH et al.: CONTRIBUTION FACTOR ESTIMATION USING ENVELOPES}

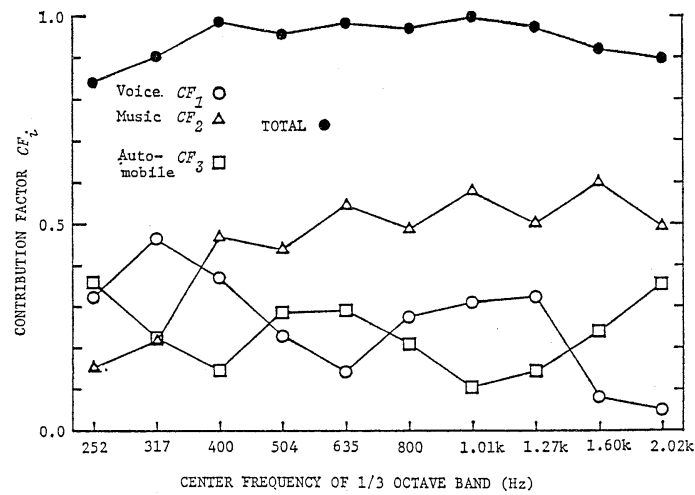

Fig. 12 Estimated contribution factor $C_{i}$ of each $1 / 3$ octave band in the open air using the filter method.

affected by the varying transmission time. The advantage of the presented method is that it is not necessary to turn on and off the source noise, nor is it necessary to use any artificial signals as a source in the measurement. Thus, with the actual noise source it becomes possible to measure the actual contribution factor necessary for noise control. Obviously, this method is inefficient in the case where the source consists of a sinusoidal wave of a single frequency, which, however, is rather a rare case. If the signal consists of a certain band width, its power fluctuates and thus the present method becomes applicable to the envelopes of the signals.

This method is easily applied to portable and special purpose systems for measuring the contribution factor in the field. Since the data sampling frequency is very low and the calculation time is not necessary to be so fast, it can be realized by small and inexpensive hardware, i.e., an analog band-pass filter bank, a low cost A/D converter and a microcomputer.

\section{ACKNOWLEDGEMENTS}

The authors wish to thank Dr. N. Tamagawa, the Director of the Research \& Development Division, Magnetic Products Group, SONY Corp., for his help and encouragement. They also wish to thank Dr. Y. Takebayashi, Mr. Y. Tabei, Mr. A. Shima (former and present students) and Prof. S. Sato of the Tohoku Institute of Technology for their discussion and advice on the preparation of this paper.

\section{REFERENCES}

1) Y. Takebayashi and K. Kido, "Effect of the shape of the time window in estimating the transfer characteristics of the acoustic system having group delay or reverberation," J. Acoust. Soc. Am., Program 96th Meeting, Vol. 64, Suppl. No. 1, S11, G8 (1978).

2) N. Aoshima, "Signal compression method applied for a measurement of sound attenuation by a barrier under wind effects," J. Acoust. Soc. Jpn. (E) 3, 105-110 (1982).

3) T. Itoh, H. Noto, T. Hirosawa, and K. Kido, “An identification of the noise sources using the envelopes of the band-limited signals-Theoretical consideration-," Tech. Rep. IECE. EA81-3, 15-22 (1981) (in Japanese).

4) M. E. Wang and M. J. Crocker, "Recent application of coherence technique for noise source identification," Inter-Noise 78 Proc. San Francisco, U.S.A., 375-382 (1978).

5) M. Abe, M. Ishigame, and K. Kido, "On the accuracy of the power spectrum estimate in the directive spectrum analyzing system by use of a moving microphone," J. Acoust. Soc. Jpn. (E) 1, 79-86 (1980).

6) W. G. Halvorsen and J. S. Bendet, "Noise source identification using coherent output power spectra," J. Sound Vib. 9, 15-24 (1975). 